\title{
A simple formula for the infrared singular part of the integrand of one-loop QCD amplitudes
}

\author{
Mohammad Assadsolimani, Sebastian Becker and Stefan Weinzierl \\ Institut für Physik, Universität Mainz, D-55099 Mainz, Germany
}

(Dated: November 2, 2018)

\begin{abstract}
We show that a well-known simple formula for the explicit infrared poles of one-loop QCD amplitudes has a corresponding simple counterpart in unintegrated form. The unintegrated formula approximates the integrand of one-loop QCD amplitudes in all soft and collinear singular regions. It thus defines a local counter-term for the infrared singularities and can be used as an ingredient for the numerical calculation of one-loop amplitudes.
\end{abstract}

PACS numbers: 11.15.Bt, 12.38.Bx, 13.87.-a

\section{INTRODUCTION}

The calculation of next-to-leading order (NLO) QCD corrections for multi-jet final states plays an important role for the experiments at the LHC. For any NLO calculation there are two parts to be calculated: the real and the virtual corrections. As far as the real corrections are concerned it has become a standard approach to first subtract out the infrared divergences [1-3] and to perform the phase space integration of the subtracted real correction term numerically by Monte Carlo techniques. The subtraction terms can be integrated and added back. The integrated form has a remarkable simple structure and predicts the infrared poles of the renormalised oneloop amplitude. The one-loop amplitude contributes to the virtual part and has in general ultraviolet, soft and collinear divergences. It involves the integration over one unconstrained momentum (the loop momentum) in $D$ dimensions. It is tempting to follow a similar path also for the virtual part: First subtract out all singular terms of ultraviolet and infrared origin from the one-loop amplitude and then perform the integration over the loop momentum together with the phase space integration in four dimensions by deforming the loop integration contour into the complex plane. The subtraction terms are then integrated analytically in $D$ dimensions and added back. Such subtraction terms have been considered in [4] and issues related to the required contour deformation have been studied in [5, 6]. The unintegrated infrared subtraction terms in [4] are determined graph by graph. This limits the efficiency of the method. Inspired by recent work on the structure of infrared singularities of multi-loop amplitudes [ 7 -9] we show in this letter that the soft and collinear subtraction terms can be formulated at the level of amplitudes, without referring to individual Feynman graphs. This is a significant simplification and opens the door to an efficient implementation based on recurrence relations [10]. The required ultraviolet subtraction terms have the form of propagator and vertex counter-terms and can be incorporated into the recurrence relations. A possible choice for the ultraviolet subtraction terms is given in ref. [4].

\section{NOTATION}

Let us consider a one-loop QCD amplitude with $n$ external partons. The full one-loop amplitude can be colour-decomposed into primitive amplitudes:

$$
\mathcal{A}^{(1)}=\sum_{j} C_{j} A_{j}^{(1)} .
$$

The colour structures are denoted by $C_{j}$ while the primitive amplitudes are denoted by $A_{j}^{(1)}$. In the colour-flow basis [11 13] the colour structures are linear combinations of monomials in Kronecker $\delta_{i j}$ 's. Primitive amplitudes are defined as a colour-stripped gauge-invariant set of Feynman diagrams with a fixed cyclic ordering of the external partons and a definite routing of the external fermions lines through the diagram 14. We have collected some information on primitive amplitudes in an appendix. It is convenient and sufficient to focus on primitive amplitudes. In the following we drop the subscript $j$ and focus on a single primitive one-loop amplitude which we denote by $A^{(1)}$. Since the cyclic ordering of the external partons is fixed, there are only $n$ different propagators occurring in the loop integral. We label the external momenta clockwise by $p_{1}, p_{2}, \ldots, p_{n}$ and define $q_{i}=p_{1}+p_{2}+\ldots+p_{i}, k_{i}=k-q_{i}$. We can write the bare primitive one-loop amplitude in Feynman gauge as

$$
\begin{aligned}
A_{\text {bare }}^{(1)} & =\int \frac{d^{D} k}{(2 \pi)^{D}} G_{\text {bare }}^{(1)} \\
G_{\text {bare }}^{(1)} & =P(k) \prod_{i=1}^{n} \frac{1}{k_{i}^{2}-m_{i}^{2}+i \delta} .
\end{aligned}
$$

$G_{\text {bare }}^{(1)}$ is the integrand of the bare one-loop amplitude. $P(k)$ is a polynomial in the loop momentum $k$. The $+i \delta$ prescription instructs us to deform - if possible - the integration contour into the complex plane to avoid the poles at $k_{i}^{2}=m_{i}^{2}$. If a deformation close to a pole is not possible, we say that the contour is pinched. In this 
letter we restrict ourselves to non-exceptional external momenta. Then the divergences of the one-loop amplitude related to a pinched contour are either due to soft or collinear partons in the loop. These divergences are regulated within dimensional regularisation by setting the number of space-time dimensions equal to $D=4-2 \varepsilon$. A primitive amplitude which has soft or collinear divergences must have at least one loop propagator which corresponds to a gluon. An amplitude which just consists of a closed fermion loop does not have any infrared divergences. We denote by $I_{g}$ the set of indices $i$, for which the propagator $i$ in the loop corresponds to a gluon. If we take the subset of diagrams which have the gluon loop propagator $i$ and if we remove from each diagram of this subset the loop propagator $i$ we obtain a set of tree diagrams. After removing multiple copies of identical diagrams this set forms a Born partial amplitude which we denote by $A_{i}^{(0)}$. After integration, the soft and collinear poles of a primitive one-loop amplitude with massless partons are given by [1, 15, 16]

$$
\begin{aligned}
& S_{\varepsilon}^{-1} \mu^{2 \varepsilon} A_{\text {bare }}^{(1)}=\frac{\alpha_{s}}{4 \pi} \frac{e^{\varepsilon \gamma_{E}}}{\Gamma(1-\varepsilon)} \sum_{i \in I_{g}} \\
& \quad\left[\frac{2}{\varepsilon^{2}}\left(\frac{-2 p_{i} \cdot p_{i+1}}{\mu^{2}}\right)^{-\varepsilon}+\frac{2}{\varepsilon}\left(S_{i}+S_{i+1}\right)\right] A_{i}^{(0)}+\mathcal{O}\left(\varepsilon^{0}\right) .
\end{aligned}
$$

We have multiplied the one-loop amplitude by $S_{\varepsilon}^{-1} \mu^{2 \varepsilon}$, where $S_{\varepsilon}=(4 \pi)^{\varepsilon} e^{-\varepsilon \gamma_{E}}$ is the typical volume factor of dimensional regularisation, $\gamma_{E}$ is Euler's constant and $\mu$ is the renormalisation scale. The constants $S_{i}$ are given by $S_{q}=S_{\bar{q}}=1$ and $S_{g}=1 / 2$, the index $i$ of $S_{i}$ refers to the external particles. The generalisation of eq. (3) to massive partons is also known.

Eq. (3) describes the infrared poles after integration over the loop momentum. In this letter we give a formula which approximates the integrand of eq. (2) in all infrared singular regions before the integration over the loop momentum. It thus defines a counter-term for the infrared singularities which is local in the loop momentum $k$ and the external momenta $p_{i}$.

\section{THE FORMULA}

In this section we present the formula for massless QCD. The extension to massive particles is discussed at the end of this letter. The infrared singular part can be written in unintegrated form as a soft and a collinear part:

$$
\begin{aligned}
G_{I R}^{(1)}= & G_{\text {soft }}^{(1)}+G_{\text {coll }}^{(1)}, \\
G_{\text {soft }}^{(1)}= & -4 \pi \alpha_{s} i \sum_{i \in I_{g}} \frac{4 p_{i} \cdot p_{i+1}}{k_{i-1}^{2} k_{i}^{2} k_{i+1}^{2}} A_{i}^{(0)}, \\
G_{\text {coll }}^{(1)}= & -4 \pi \alpha_{s} i \sum_{i \in I_{g}}(-2)\left(\frac{S_{i} g_{U V}\left(k_{i-1}^{2}, k_{i}^{2}, \mu_{c}^{2}\right)}{k_{i-1}^{2} k_{i}^{2}}\right. \\
& \left.+\frac{S_{i+1} g_{U V}\left(k_{i}^{2}, k_{i+1}^{2}, \mu_{c}^{2}\right)}{k_{i}^{2} k_{i+1}^{2}}\right) A_{i}^{(0)} .
\end{aligned}
$$

The Born partial amplitude $A_{i}^{(0)}$ depends on the external momenta, but not on the loop momentum. The function $g_{U V}$ ensures a regular behaviour of the collinear term in the ultraviolet region. A possible choice is [4]

$$
g_{U V}\left(k_{i-1}^{2}, k_{i}^{2}, \mu_{c}^{2}\right)=\frac{1}{2}\left(\frac{-\mu_{c}^{2}}{k_{i-1}^{2}-\mu_{c}^{2}}+\frac{-\mu_{c}^{2}}{k_{i}^{2}-\mu_{c}^{2}}\right) .
$$

$\mu_{c}$ is an arbitrary scale. Integrating the soft and the collinear part we obtain

$$
\begin{aligned}
& S_{\varepsilon}^{-1} \mu^{2 \varepsilon} \int \frac{d^{D} k}{(2 \pi)^{D}} G_{\text {soft }}^{(1)}=\frac{\alpha_{s}}{4 \pi} \frac{e^{\varepsilon \gamma_{E}}}{\Gamma(1-\varepsilon)} \\
& \quad \times \sum_{i \in I_{g}} \frac{2}{\varepsilon^{2}}\left(\frac{-2 p_{i} \cdot p_{i+1}}{\mu^{2}}\right)^{-\varepsilon} A_{i}^{(0)}+\mathcal{O}(\varepsilon), \\
& S_{\varepsilon}^{-1} \mu^{2 \varepsilon} \int \frac{d^{D} k}{(2 \pi)^{D}} G_{\text {coll }}^{(1)}=\frac{\alpha_{s}}{4 \pi} \frac{e^{\varepsilon \gamma_{E}}}{\Gamma(1-\varepsilon)} \\
& \quad \times \sum_{i \in I_{g}}\left(S_{i}+S_{i+1}\right)\left(\frac{\mu_{c}^{2}}{\mu^{2}}\right)^{-\varepsilon}\left(\frac{2}{\varepsilon}+2\right) A_{i}^{(0)}+\mathcal{O}(\varepsilon) .
\end{aligned}
$$

Equation (44) is the main result of this letter. This formula approximates the integrand of a primitive one-loop QCD amplitude in all soft and collinear limits. The approximation is given by simple scalar two- and threepoint functions, multiplied by a Born partial amplitude. One easily observes that the integrated form in eq. (6) agrees in the pole terms with eq. (3).

\section{PROOF OF THE FORMULA}

We first review briefly under which conditions infrared singularities occur in an individual Feynman diagram [4, 17, 18]: Soft singularities occur when a massless particle is exchanged between two on-shell particles. With the notation as in eq. (2) this corresponds to the case

$$
m_{i}=0, \quad p_{i}^{2}=m_{i-1}^{2}, \quad p_{i+1}^{2}=m_{i+1}^{2} .
$$

In that case the propagators $(i-1), i$ and $(i+1)$ are on-shell. The singularity comes from the integration region $k \sim q_{i}$. A collinear singularity occurs if a massless external on-shell particle is attached to two massless 
propagators. This corresponds to

$$
p_{i}^{2}=0, \quad m_{i-1}=0, \quad m_{i}=0 .
$$

In that case the propagators $(i-1)$ and $i$ are on-shell. The singularity comes from the integration region $k \sim q_{i}-x p_{i}$, where $x$ is a real variable between 0 and 1 . In order to proof eq. (4) we consider now massless QCD amplitudes.

The soft subtraction term is derived as follows: In the case where gluon $i$ is soft, the corresponding propagator goes on-shell and we may replace in all Feynman diagrams which have propagator $i$ the metric tensor $g_{\mu \nu}$ of this propagator by a polarisation sum and gauge terms:

$$
\frac{-i g^{\mu \nu}}{k_{i}^{2}} \rightarrow \frac{i}{k_{i}^{2}}\left(d^{\mu \nu}\left(k_{i}^{b}, n\right)-2 \frac{k_{i}^{b \mu} n^{\nu}+n^{\mu} k_{i}^{b \nu}}{2 k_{i}^{b} \cdot n}\right) .
$$

Here $k_{i}^{b}$ denotes the on-shell limit of $k_{i}$ and $d^{\mu \nu}$ denotes the sum over the physical polarisations:

$$
\begin{aligned}
d^{\mu \nu}(k, n) & =\sum_{\lambda} \varepsilon_{\lambda}^{\mu}(k, n) \varepsilon_{-\lambda}^{\nu}(k, n) \\
& =-g^{\mu \nu}+2 \frac{k^{\mu} n^{\nu}+n^{\mu} k^{\nu}}{2 k \cdot n} .
\end{aligned}
$$

$n^{\mu}$ is a light-like reference vector. We note that selfenergy diagrams are not singular in the soft limit, therefore adding them to the loop diagrams will not change the soft limit. With the inclusion of the self-energy diagrams and a corresponding replacement as in eq. (9) the contribution from the polarisation sum in eq. (9) makes up a tree-level partial amplitude, where two gluons with momenta $k_{i}^{b}$ and $-k_{i}^{b}$ have been inserted between the external legs $i$ and $i+1$. In the soft limit this tree-level partial amplitude is given by two eikonal factors times the tree-level partial amplitude without these two additional gluons:

$$
\left(i g \frac{p_{i}^{\mu}}{p_{i} \cdot k_{i}^{b}}\right) g_{\mu \nu}\left(i g \frac{p_{i+1}^{\nu}}{p_{i+1} \cdot\left(-k_{i}^{b}\right)}\right) A_{i}^{(0)} .
$$

In the soft limit we may replace $2 p_{i} \cdot k_{i}^{b}$ by $k_{i-1}^{2}$ and $2 p_{i+1}$. $\left(-k_{i}^{b}\right)$ by $k_{i+1}^{2}$. Eq. (11) then leads to the soft part of eq. (44). The terms with $k_{i}^{b \mu} n^{\nu}$ and $n^{\mu} k_{i}^{b \nu}$ in eq. (9) vanish for the sum of all diagrams due to gauge invariance.

The collinear subtraction term is derived as follows: We have to consider diagrams, where two adjacent propagators in the loop go on-shell with a massless external leg in between. The cases where an external gluons splits into a ghost-antighost-pair or into a quark-antiquark-pair are in the collinear limit not singular enough to yield a divergence after integration. Therefore we are left with the case where an external quark splits into a quark-gluon pair and the case where an external gluon splits into two gluons. Let us first consider the $q \rightarrow q g$ splitting. In Feynman gauge one can show that only the longitudinal polarisation of the gluon contributes to the collinear limit. The same holds true for the $g \rightarrow g g$ splitting. In this case the collinear limit receives contributions when one of the two gluons in the loop carries a longitudinal polarisation (but not both). The external gluon has of course physical transverse polarisation. We can now use gauge-invariance to turn the sum of all diagrams with a collinear singularity in the propagators $(i-1)$ and $i$ into the simple form of eq. (4). The contraction of a longitudinal polarisation into a gauge-invariant set of diagrams yields zero. The set of collinear divergent diagrams forms an almost gauge-invariant set of diagrams. There is only one diagram missing, where the longitudinal polarised gluon couples directly to the other parton. This is a self-energy insertion on an external line, which by definition is absent from the amputated one-loop amplitude. We can now turn the argument around and replace the sum of collinear singular diagrams by the negative of the self-energy insertion on the external line. The self-energy insertions on the external lines introduce a spurious $1 / p_{i^{-}}^{2}$ singularity. In order to calculate the singular part of the self-energies we regulate this spurious singularity by allowing $p_{i}^{2}$ slightly off-shell, but keeping $k_{i-1}$ and $k_{i}$ onshell and imposing momentum conservation. We can use the same parametrisation as in the real emission case:

$$
\begin{aligned}
& k_{i-1}=x p+k_{\perp}-\frac{k_{\perp}^{2}}{x} \frac{n}{(2 p \cdot n)}, \\
& -k_{i}=(1-x) p-k_{\perp}-\frac{k_{\perp}^{2}}{(1-x)} \frac{n}{(2 p \cdot n)},
\end{aligned}
$$

with $p^{2}=n^{2}=0$ and $2 p \cdot k_{\perp}=2 n \cdot k_{\perp}=0$. The singular part of the self-energies with one longitudinal polarised gluon is proportional to

$$
\begin{aligned}
& P_{q \rightarrow q g}^{l o n g}=-\frac{2}{2 k_{i-1} \cdot k_{i}}\left(-\frac{2}{1-x}+2\right) \not p \\
& P_{g \rightarrow g g}^{l o n g}=-\frac{2}{2 k_{i-1} \cdot k_{i}}\left(-\frac{2}{x}-\frac{2}{1-x}+2\right) d^{\mu \nu}(p, n) .
\end{aligned}
$$

The terms with $2 / x$ and $2 /(1-x)$ correspond to soft singularities and have already been subtracted out with the soft subtraction term $G_{\text {soft }}^{(1)}$. In the collinear limit we therefore just have to subtract out the terms, which are non-singular in the soft limit. These terms are independent of $x$ and lead to the collinear part of eq. (4).

A few remarks are in order: Eq. (3) is usually stated for the renormalised one-loop amplitude.

$$
\begin{aligned}
\mathcal{A}_{\text {ren }}^{(1)}= & \frac{\alpha_{s}}{4 \pi} \frac{e^{\varepsilon \gamma_{E}}}{\Gamma(1-\varepsilon)}\left[\sum_{\text {pairs }(i, j)} \frac{2 \mathbf{T}_{i} \mathbf{T}_{j}}{\varepsilon^{2}}\left(\frac{-2 p_{i} \cdot p_{i+1}}{\mu^{2}}\right)^{-\varepsilon}\right. \\
& \left.-\sum_{i} \frac{\gamma_{i}}{\varepsilon}\right] \mathcal{A}^{(0)}+\mathcal{O}\left(\varepsilon^{0}\right) .
\end{aligned}
$$

The colour charge operators are given by $\mathbf{T}_{q}=T_{i j}^{a}$, $\mathbf{T}_{\bar{q}}=-T_{j i}^{a}$ and $\mathbf{T}_{g}=i f^{b a c}$ for final state particles. 
The constants $\gamma_{i}$ are given by $\gamma_{q}=\gamma_{\bar{q}}=3 C_{F} / 2$ and $\gamma_{g}=\beta_{0} / 2$ with $\beta_{0}=11 C_{A} / 3-4 T_{R} N_{f} / 3$. The renormalised one-loop amplitude with $n_{g}$ gluons, $n_{q}$ quarks and $n_{\bar{q}}$ antiquarks is related to the bare amplitude by

$$
\begin{aligned}
\mathcal{A}_{\text {ren }}\left(p_{1}, \ldots, p_{n}, \alpha_{s}\right)= & \left(Z_{2}^{1 / 2}\right)^{n_{q}+n_{\bar{q}}}\left(Z_{3}^{1 / 2}\right)^{n_{g}} \\
& \times \mathcal{A}_{\text {bare }}\left(p_{1}, \ldots, p_{n}, Z_{g}^{2} S_{\varepsilon}^{-1} \mu^{2 \varepsilon} \alpha_{s}\right) .
\end{aligned}
$$

$Z_{g}$ is coupling renormalisation constant

$$
Z_{g}=1+\frac{\alpha_{s}}{4 \pi}\left(-\frac{\beta_{0}}{2}\right) \frac{1}{\varepsilon}+\mathcal{O}\left(\alpha_{s}^{2}\right) .
$$

$Z_{2}$ is the quark field renormalisation constant and $Z_{3}$ is the gluon field renormalisation constant. The LSZ reduction formula instructs us to take for the field renormalisation constants the residue of the propagators at the pole. In dimensional regularisation this residue is 1 for massless particles and therefore the field renormalisation constants are often omitted from eq. (15). However $Z_{2}=Z_{3}=1$ is due to a cancellation between ultraviolet and infrared divergences. In Feynman gauge we have

$$
\begin{aligned}
& Z_{2}=1+\frac{\alpha_{s}}{4 \pi} C_{F}\left(\frac{1}{\varepsilon_{I R}}-\frac{1}{\varepsilon_{U V}}\right)+\mathcal{O}\left(\alpha_{s}^{2}\right) \\
& Z_{3}=1+\frac{\alpha_{s}}{4 \pi}\left(2 C_{A}-\beta_{0}\right)\left(\frac{1}{\varepsilon_{I R}}-\frac{1}{\varepsilon_{U V}}\right)+\mathcal{O}\left(\alpha_{s}^{2}\right) .
\end{aligned}
$$

Here we indicated explicitly the origin of the $1 / \varepsilon$-poles. Using $Z_{2}=Z_{3}=1$ mixes therefore poles of ultraviolet and infrared origin. There are different variants of dimensional regularisation: conventional dimensional regularisation, the 't Hooft-Veltman scheme and fourdimensional schemes. The soft and collinear subtraction terms in eq. (4) are independent of the variant of dimensional regularisation. The infrared poles obtained from the phase space integration of the real emission amplitude depend on the other hand on the scheme of dimensional regularisation. Again the solution is due to the field renormalisation constants which mix schemedependent terms of ultraviolet and infrared origin [19]. The scheme-dependence of the bare one-loop amplitude is entirely of ultraviolet origin.

\section{GENERALISATION TO MASSIVE PARTONS}

In this section we present the generalisation to massive QCD. This is in particular relevant to top quark physics. There are only a few modifications necessary with respect to the massless case. The modification for the unintegrated soft subtraction term is straightforward:

$$
\begin{aligned}
& G_{\text {soft }}^{(1)}= \\
& \quad-4 \pi \alpha_{s} i \sum_{i \in I_{g}} \frac{4 p_{i} \cdot p_{i+1}}{\left(k_{i-1}^{2}-m_{i-1}^{2}\right) k_{i}^{2}\left(k_{i+1}^{2}-m_{i+1}^{2}\right)} A_{i}^{(0)} .
\end{aligned}
$$

As before, the sum runs over all particles in the loop which are gluons. Integrating the soft subtraction term we have to distinguish whether the masses $m_{i-1}$ and $m_{i+1}$ are zero or not. The result can be written as

$$
\begin{aligned}
& S_{\varepsilon}^{-1} \mu^{2 \varepsilon} \int \frac{d^{D} k}{(2 \pi)^{D}} G_{\text {soft }}^{(1)}=\frac{\alpha_{s}}{4 \pi} \frac{e^{\varepsilon \gamma_{E}}}{\Gamma(1-\varepsilon)} \sum_{i \in I_{g}} \\
& \quad \times C\left(\left(p_{i}+p_{i+1}\right)^{2}, m_{i-1}^{2}, m_{i+1}^{2}, \mu^{2}\right) A_{i}^{(0)}+\mathcal{O}(\varepsilon),
\end{aligned}
$$

where the function $C\left(s, m_{1}^{2}, m_{2}^{2}, \mu^{2}\right)$ is given for the four different cases by [18, 20]

$$
\begin{gathered}
C\left(s, 0,0, \mu^{2}\right)=\frac{2}{\varepsilon^{2}}\left(\frac{-s}{\mu^{2}}\right)^{-\varepsilon}, \\
C\left(s, 0, m^{2}, \mu^{2}\right)=C\left(s, m^{2}, 0, \mu^{2}\right), \\
C\left(s, m^{2}, 0, \mu^{2}\right)=\left(\frac{m^{2}}{\mu^{2}}\right)^{-\varepsilon}\left[\frac{1}{\varepsilon^{2}}+\frac{2}{\varepsilon} \ln \left(\frac{m^{2}}{m^{2}-s}\right)\right. \\
\left.+\frac{\pi^{2}}{6}+\ln ^{2}\left(\frac{m^{2}}{m^{2}-s}\right)-2 \operatorname{Li}_{2}\left(\frac{-s}{m^{2}-s}\right)\right], \\
C\left(s, m_{1}^{2}, m_{2}^{2}, \mu^{2}\right)=\frac{2 x\left(s-m_{1}^{2}-m_{2}^{2}\right)}{m_{1} m_{2}\left(1-x^{2}\right)}\left\{\left[-\frac{1}{\varepsilon}-\frac{1}{2} \ln (x)\right.\right. \\
\left.+2 \ln \left(1-x^{2}\right)+\ln \left(\frac{m_{1} m_{2}}{\mu^{2}}\right)\right] \ln (x)-\frac{\pi^{2}}{6}+\operatorname{Li}_{2}\left(x^{2}\right) \\
\left.+\frac{1}{2} \ln ^{2}\left(\frac{m_{1}}{m_{2}}\right)+\operatorname{Li}_{2}\left(1-x \frac{m_{1}}{m_{2}}\right)+\operatorname{Li}_{2}\left(1-x \frac{m_{2}}{m_{1}}\right)\right\},
\end{gathered}
$$

with

$$
x=-\frac{1-\chi}{1+\chi}, \quad \chi=\sqrt{1-\frac{4 m_{1} m_{2}}{s-\left(m_{1}-m_{2}\right)^{2}}} .
$$

The modification for the collinear subtraction term is even simpler: There is no collinear singularity if an external quark or antiquark is massive. It suffices therefore to define $S_{Q}=S_{\bar{Q}}=0$ for a massive quark or antiquark.

\section{CONCLUSIONS}

In this letter we have shown that the infrared singular part of the integrand of a primitive one-loop QCD amplitude is given by simple scalar two- and three-point functions, multiplied by a Born partial amplitude. The immediate application is to use this form as a subtraction term for the numerical integration of a one-loop amplitude. In view of refs. [7 -9] we do not exclude the possibility that this method generalises to higher loops.

\section{Primitive amplitudes}

In this appendix we include a brief summary on primitive one-loop amplitudes. In order to construct a primitive one-loop amplitude one starts to draw all possible 


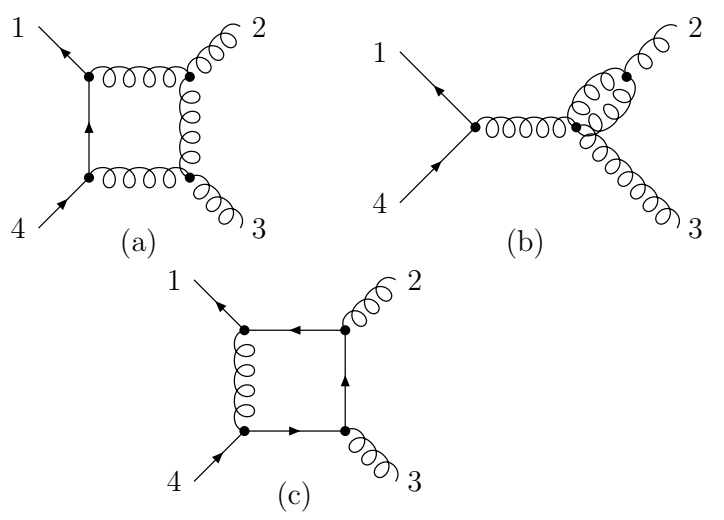

FIG. 1: Examples of diagrams: Diagrams (a) and (b) contribute to the left-moving primitive amplitude, while diagram (c) contributes to the right-moving amplitude.

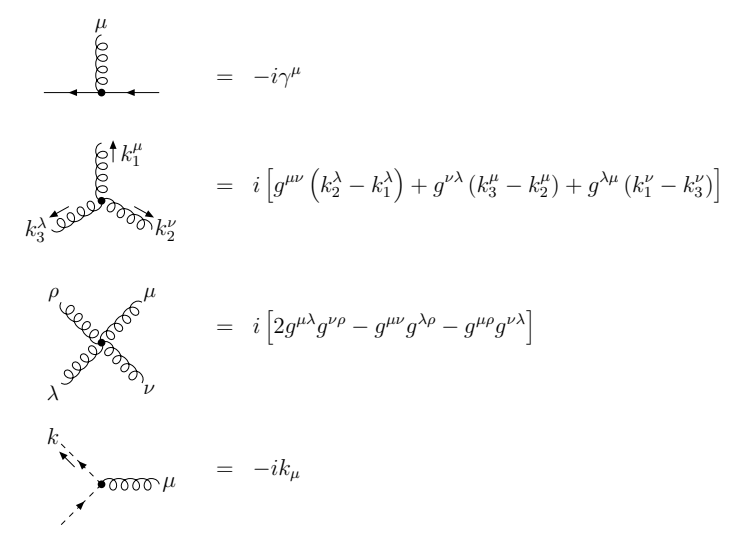

FIG. 2: Colour-ordered Feynman rules.

planar one-loop diagrams with a fixed cyclic order of the external legs, subject to the constraint that each fermion line is either only left-moving or only right-moving. We call a fermion line "left-moving", if following the arrow of the fermion line the loop is to the right. In an amplitude with $r$ external quark-antiquark pairs $p$ of these pairs can be left-moving The remaining $(r-p)$ pairs are then rightmoving. As an example we consider primitive $\bar{q} g g q-$ amplitudes with the cyclic order $\left(1_{\bar{q}}, 2 g, 3_{g}, 4_{q}\right)$. Fig. 1 shows some diagrams as examples. Diagrams (a) and (b) contribute to the primitive amplitude, where the quark line is left-moving, diagram (c) however contributed to the primitive amplitude, where the quark line is rightmoving. We would like to point out that diagram (b) has to be included. In an analytic calculation this diagram is often discarded, because it yields zero within dimensional regularisation. However this zero is obtained from a cancellation between an ultraviolet divergence and a collinear divergence. In a numerical calculation we have to keep this diagram in order not to spoil the local structure.
A diagram is translated to a formula with the help of the following Feynman rules: The propagators for a quark, gluon and ghost particle are

$$
i \frac{\not k+m}{k^{2}-m^{2}}, \quad \frac{-i g^{\mu \nu}}{k^{2}}, \quad \frac{i}{k^{2}}
$$

respectively. The Feynman rules for the vertices are listed in fig. 2.

In the main part of the paper we made use of the Ward identity for cyclic ordered amplitudes. The Ward identity states if we replace in the amplitude the polarisation vector of one external gluon by its momentum then we obtain zero. A proof can be found in the textbook by Peskin and Schroeder [21].

[1] S. Catani and M. H. Seymour, Nucl. Phys. B485, 291 (1997), hep-ph/9605323.

[2] L. Phaf and S. Weinzierl, JHEP 04, 006 (2001), hep$\mathrm{ph} / 0102207$.

[3] S. Catani, S. Dittmaier, M. H. Seymour, and Z. Trocsanyi, Nucl. Phys. B627, 189 (2002), hep-ph/0201036.

[4] Z. Nagy and D. E. Soper, JHEP 09, 055 (2003), hep$\mathrm{ph} / 0308127$.

[5] Z. Nagy and D. E. Soper, Phys. Rev. D74, 093006 (2006), hep-ph/0610028.

[6] W. Gong, Z. Nagy, and D. E. Soper, Phys. Rev. D79, 033005 (2009), 0812.3686.

[7] T. Becher and M. Neubert, Phys. Rev. Lett. 102, 162001 (2009), 0901.0722.

[8] E. Gardi and L. Magnea, JHEP 03, 079 (2009), 0901.1091.

[9] A. Ferroglia, M. Neubert, B. D. Pecjak, and L. L. Yang (2009), 0907.4791.

[10] F. A. Berends and W. T. Giele, Nucl. Phys. B306, 759 (1988).

[11] G. 't Hooft, Nucl. Phys. B72, 461 (1974).

[12] F. Maltoni, K. Paul, T. Stelzer, and S. Willenbrock, Phys. Rev. D67, 014026 (2003), hep-ph/0209271.

[13] S. Weinzierl, Eur. Phys. J. C45, 745 (2006), hep$\mathrm{ph} / 0510157$.

[14] Z. Bern, L. J. Dixon, and D. A. Kosower, Nucl. Phys. B437, 259 (1995), hep-ph/9409393.

[15] W. T. Giele and E. W. N. Glover, Phys. Rev. D46, 1980 (1992).

[16] Z. Kunszt, A. Signer, and Z. Trocsanyi, Nucl. Phys. B420, 550 (1994), hep-ph/9401294.

[17] T. Kinoshita, J. Math. Phys. 3, 650 (1962).

[18] S. Dittmaier, Nucl. Phys. B675, 447 (2003), hep$\mathrm{ph} / 0308246$.

[19] B. W. Harris, E. Laenen, L. Phaf, Z. Sullivan, and S. Weinzierl, Phys. Rev. D66, 054024 (2002), hep$\mathrm{ph} / 0207055$.

[20] R. K. Ellis and G. Zanderighi, JHEP 02, 002 (2008), 0712.1851.

[21] M. E. Peskin and D. V. Schroeder, An Introduction to Quantum Field Theory (Perseus Books, 1995). 